\title{
miR-128 inhibits telomerase activity by targeting TERT mRNA
}

\author{
Herlinda Guzman ${ }^{1, *}$, Katie Sanders ${ }^{1, *}$, Adam Idica ${ }^{1}$, Aurore Bochnakian ${ }^{1}$, Douglas \\ Jury ${ }^{1}$, Iben Daugaard ${ }^{1}$, Dimitrios G Zisoulis ${ }^{1}$ and Irene Munk Pedersen ${ }^{1}$ \\ ${ }^{1}$ Department of Molecular Biology and Biochemistry, Francisco J. Ayala School of Biological Sciences, University of California, \\ Irvine 92697-3900, CA, USA \\ *These authors contributed equally to this work
}

Correspondence to: Irene Munk Pedersen, email: imp@uci.edu

Keywords: telomerase; miR-128; miR; TERT; cancer

Received: November 30, $2017 \quad$ Accepted: January 09, $2018 \quad$ Published: January 19, 2018

Copyright: Guzman et al. This is an open-access article distributed under the terms of the Creative Commons Attribution License 3.0 (CC BY 3.0), which permits unrestricted use, distribution, and reproduction in any medium, provided the original author and source are credited.

\section{ABSTRACT}

Telomerase is a unique cellular reverse transcriptase (RT) essential for maintaining telomere stability and required for the unlimited proliferation of cancer cells. The limiting determinant of telomerase activity is the catalytic component TERT, and TERT expression is closely correlated with telomerase activity and cancer initiation and disease progression. For this reason the regulation of TERT levels in the cell is of great importance. microRNAs (miRs) function as an additional regulatory level in cells, crucial for defining expression boundaries, proper cell fate decisions, cell cycle control, genome integrity, cell death and metastasis. We performed an anti-miR library screen to identity novel miRs, which participate in the control of telomerase. We identified the tumor suppressor $\mathrm{miR}(\mathrm{miR}-128)$ as a novel endogenous telomerase inhibitor and determined that miR-128 significantly reduces the mRNA and protein levels of Tert in a panel of cancer cell lines. We further evaluated the mechanism by which miR-128 regulates TERT and demonstrated that miR-128 interacts directly with the coding sequence of TERT MRNA in both HeLa cells and teratoma cells. Interestingly, the functional miR-128 binding site in TERT MRNA, is conserved between TERT and the other cellular reverse transcriptase encoded by Long Interspersed Elements-1 (LINE-1 or L1), which can also contribute to the oncogenic phenotype of cancer. This finding supports the novel idea that miRs may function in parallel pathways to inhibit tumorigenesis, by regulating a group of enzymes (such as RT) by targeting conserved binding sites in the coding region of both enzymes.

\section{INTRODUCTION}

Limitless replicative potential is considered a hallmark of cancer, which is achieved by an inappropriate reactivation of the essential enzyme telomerase $[1,2]$. Telomerase maintains telomere integrity by adding the six-nucleotide repeat sequence, 5'-TTAGGG, to the ends of the chromosomes using its internal template RNA component (TERC) and reverse transcriptase protein component (TERT), thus counteracting the telomere shortening that naturally occurs during DNA replication
[3-6]. Telomerase is not expressed in most adult somatic cells and the telomeres are therefore progressively shortened with each round of replication, which ultimately leads to replicative senescence and thus a finite replicative potential. In contrast, stem cells and cancer cells have high telomerase activity. In fact, TERT has been shown to be overexpressed in almost $90 \%$ of all human malignancies, but although there is evidence that mutations in the TERT promoter lead to enhanced TERT expression, the mechanisms by which telomerase is reactivated is still poorly understood [7-11]. 
microRNAs (miRs) are a class of small $(\sim 22$ nucleotide) non-coding RNAs, which are loaded onto Argonaute (Ago) proteins forming the miR-induced silencing complex (miRISC) and function as posttranscriptional regulators of gene expression by inducing mRNA instability or translational repression [12]. More than $60 \%$ of all protein-coding genes are believed to be subjects of miR regulation and alterations in miR expression can therefore have dire consequences and contribute to the development of a wide variety of human diseases, including cancer [12-17]. Depending on their role in carcinogenesis, miRs can generally be divided into oncogenic miRs (oncomiRs) or tumor suppressor miRs that promote or inhibit tumor development and progression, respectively [17-19]. miRs are suspected to be implicated in telomerase reactivation and a subset has been shown to affect telomerase activity directly, e.g. by inducing TERT expression. For instance, miR-138 has been reported to function as a direct regulator of TERT expression in thyroid carcinoma and at least 5 additional tumor suppressor miRs (let-7g, miR-133a, miR-342, miR-491 and miR-541) have been shown to be capable of regulating TERT expression through direct interaction with TERT mRNA [20,21].

Aberrant expression of miR-128 is a frequent observation in human malignancies, but depending on the tumor type, it has been shown to be capable of acting both as an oncomiR and a tumor suppressor miR [22, 23]. Most studies have, however, found it to act as a tumor suppressor and downregulation has been documented in a long list of human malignancies, including glioma, prostate, head and neck, lung and colorectal cancer, where it has been shown to function as an inhibitor of cancer cell growth and metastasis [22, 24-28]. We recently demonstrated that miR-128 regulates another cellular reverse transcriptase, namely the Long-Interspersed Element-1 (LINE-1 or L1) by directly interacting with ORF2 L1 RNA, which encodes L1 RT [29] and indirectly by regulating a required host factor (Transportin-1 or TNPO1) needed for nuclear import of L1-RNP complexes [29, 30]. De-repression of L1 elements have been demonstrated to function as driver mutations during tumor initiation, as well as during tumor progression [31-37].

In this study, we identified miR-128 as a regulator of telomerase activity in an anti-miR library screen, demonstrating that endogenously expressed miR-128 inhibits telomerase activity in HeLa cells. Furthermore, we found that overexpression of miR-128 decreased TERT mRNA and protein levels and miR-128 depletion enhanced the levels of TERT mRNA and protein, relative to controls, in a panel of cell lines. Finally, we demonstrate that miR-128 regulates telomerase activity by directly targeting two sites in the coding region of TERT mRNA. These findings show that tumor suppressor miR-128 also effect the oncogenic phenotype of cancer cells by regulating telomerase.

\section{RESULTS}

\section{Identification of miR-128 as a regulator of telomerase}

We have recently established that miRs (miR-128) can repress the activity of key enzymes in our cells, such as reverse transcriptase (RT) encoded by transposable elements (long-Interspersed element-1, LINE-1 or L1) [29]. With this in mind we turned our attention to the most famous RT in human cells - telomerase - an enzyme which plays a crucial role in cancer, stem cells and aging [7-9].

We developed a lentiviral anti-miR screen as a way to identify miRs that play a regulatory role of telomerase in HeLa cells. In brief, we transduced HeLa cells with an anti-miR library encoding conserved and well-characterized miRs that neutralize the corresponding endogenously expressed miR in the transduced $\mathrm{HeLa}$ cells. The lentivirus also encodes copGFP (green) and puromycin for positive selection of transduced cells. This approach favors a physiologically relevant response by avoiding potential artifacts resulting from ectopic overexpression in cells, which does not normally express a specific miR. Following transduction of the antimiR library or control miRs, we performed single cell dilutions into 96-well plates and performed a qPCR based functional assay of telomerase activity, using the telomeric repeat amplification protocol (q-TRAP) [9]. The q-TRAP assay involves extension of an oligonucleotide through telomerase-mediated enzymatic addition of telomeric DNA repeats and subsequent PCR amplification of the extension products and serves as a great high throughput functional assay for telomerase activity in cells (Figure 1A). Shown in Figure 1B is a panel of miR controls (Controls) and one positive control, tested in parallel with samples, which had been transduced with the anti-miR library (Samples) and plated in single cell dilution and analyzed for telomerase activity.

We identified anti-miR-128 as an anti-miR, which significantly de-repress telomeric repeat amplification, as determined by q-TRAP analysis, (Figure 1B). Of note, miR-128 was cloned out from 4 independent clones. We repeated the assays with a subset of miR-modulated HeLa samples and verified that anti-miR-128 enhances telomerase activity (Figure 1C). To test the specificity of the anti-miR-128 effect on telomerase activity, we generated high titer miR-128, anti-miR-128 and control miR lentiviruses, transduced HeLa cells and selected for puromycin resistance. The miR-128 cell line panel was then evaluated for telomerase activity as described using the q-TRAP assay. As expected anti-miR-128 significantly increased HeLa cell telomerase activity, compared to cells expressing endogenous miR-128 (Control) (Figure 1D). In contrast, miR-128 significantly reduced the level of telomeric repeat amplification, relative to miR controls 
(Figure 1D). These experiments suggest that miR-128 regulates telomerase activity in HeLa cells.

\section{miR-128 reduces TERT $m R N A$ and protein levels}

We next wished to characterize the mechanism by which miR-128 regulates telomerase activity. First, we performed RT-qPCR analysis of HeLa cells in order to determine if miR-128 acts by regulating the amount of TERT mRNA. Induced miR-128 expression significantly decreased TERT mRNA levels, relative to miR controls, whereas miR-128 neutralization by anti-miR-128 resulted in an enhanced amount of TERT mRNA, compared to HeLa miR control samples (Figure 2A). In addition, miR-128 overexpression resulted in significant degreased TERT mRNA levels in a teratoma cell line (Tera-1 or Tera) and in two iPSC cell lines, relative to miR controls (Supplementary Figure 1). anti-miR-128 showed a similar tendency as seen in HeLa cells, though less substantial in the iPS cell lines (Supplementary Figure 1).

We next examined the potency of miR-128 at regulating Tert protein levels in HeLa cells. We determined that miR-128 significantly decreased Tert
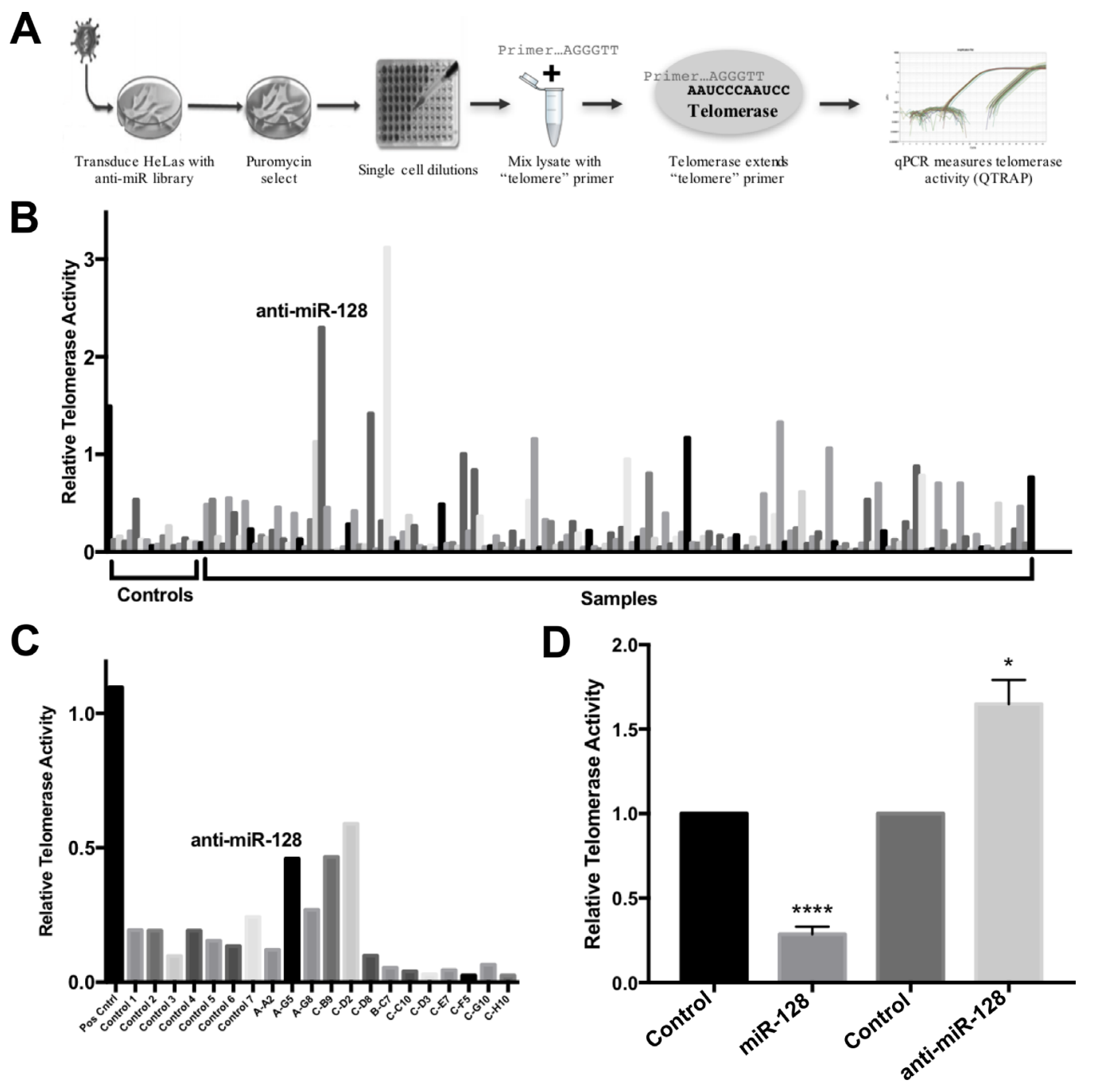

Figure 1: Identification of miR-128 as an inhibitor of telomerase activity. (A) Schematic of the anti-miR library screen to identify miRs that regulate telomerase activity. HeLa cells were transduced using a lentiviral-based, miR-neutralizing shRNA library, selected for puromycin resistance and clonally expanded. Each well represents the neutralization of a single endogenously expressed miR. Cells were then subjected to quantitative PCR-based TRAP assay (q-TRAP) analysis using the quantitative telomeric repeat amplification protocol. (B) Relative telomerase activity of HeLa cells after transduction with lentiviral miR-neutralizing anti-miR library, selection, and clonal expansion as measured by quantitative telomeric repeat amplification protocol (q-TRAP) as shown. Shown is a panel of miR controls (Controls), relative to samples transduced with the anti-miR library (Samples). (C) Secondary measurement of relative telomerase activity in select samples of anti-miR library-expressing HeLa cells, as described for Figure 1B. (D) Single high-titer miR-control, miR128 and anti-miR-128 lentiviruses were generated and stable miR-modulated HeLa cells were assayed for relative telomerase activity measured by q-TRAP analysis. Results shown as percent change $\pm \operatorname{SEM}\left(n=3\right.$, independent biological replicates, $\left.{ }^{*} p<0.05,{ }^{* * * *} p<0.0001\right)$. 
protein levels, compared to miR controls. In contrast, anti-miR-128 significantly enhanced Tert protein levels, relative to cells expressing miR control (Figure 2B). In addition, analysis of Tert protein levels and localization was analyzed by confocal analysis, demonstrating that miR-128 significantly reduces nuclear staining of Tert protein in HeLa cells (seen as red single channel Tert staining and pink staining in overlay images with DAPI stained nuclei), relative to miR control HeLa cells (Figure 2C). As expected, anti-miR-128 significantly enhanced Tert protein nuclear expression, compared to miR control HeLa cells (Figure 2C). Finally, we verified that miR128 significantly regulates Tert protein levels in a small panel of cancer cell lines, including lung cancer (A549), colon cancer (SW620) and pancreatic cancer (PANC1), demonstrating that the effect of miR-128 on Tert is not limited to HeLa cells (Figure 2D). Taken together this data demonstrate that miR-128 regulates Tert expression both at the mRNA and protein levels in different cell types.

\section{miR-128 interacts with the coding sequence of TERT mRNA.}

miR-128 could potentially regulate telomerase activity by directly targeting TERT mRNA, or by regulating expression of other proteins that regulate telomerase, or both. In parallel with this work our laboratory have been investigating the mechanisms by which miR-128 regulate L1-induced mutagenesis, including the repression of L1 RT activity. We have demonstrated that miR-128 repress L1 retrotransposition and genomic integration by directly targeting the L1 RNA, in a similar fashion as when miRs function as an anti-viral defense mechanism in human cells, limiting viral replication of RNA virus [29, 38, 39]. We surprisingly determined that miR-128 targets L1 RNA by binding directly through an imperfect seed match to L1 RNA in the coding sequence (CDS) of ORF2, which encodes the L1 endonuclease and reverse transcriptase (RT). With our new finding that miR-128 can also repress telomerase activity in a functional assay (q-TRAP) and by regulating TERT mRNA and protein levels (Figure 2), we hypothesized that miR-128 might be binding to a shared conserved site between L1 RT and TERT mRNA. We aligned the mRNA sequences of the two cellular RTs (L1 and TERT) and determined that the functional miR-128 binding site in L1 is, in fact, present at two locations in the CDS of TERT mRNA (Figure 3A and 3B). To test if the two noncanonical miR-128 seed sites are functional, we generated TERT CDS luciferase constructs either encoding the wildtype (WT) TERT binding Site \#1 or binding Site \#2. In addition, we generated a $23 \mathrm{nt}$ perfect miR-128 match
A

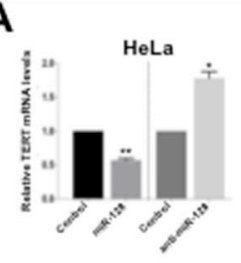

B
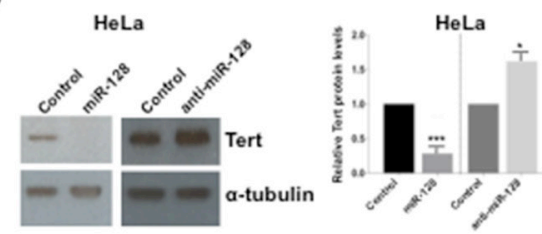

D

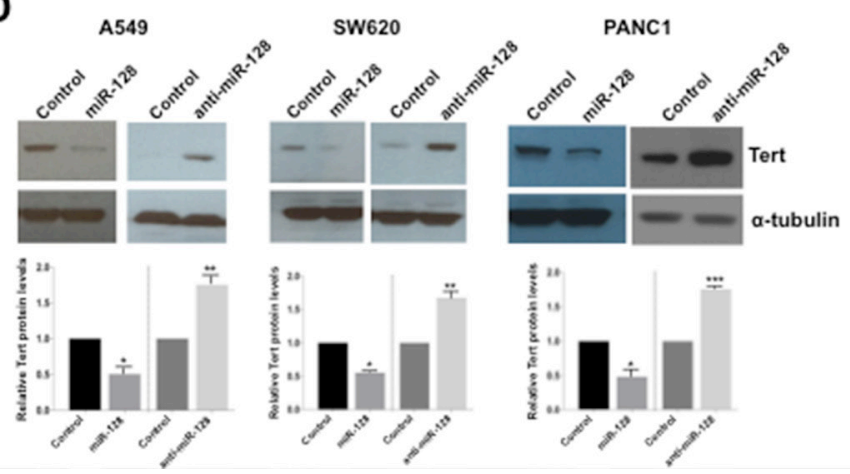

C
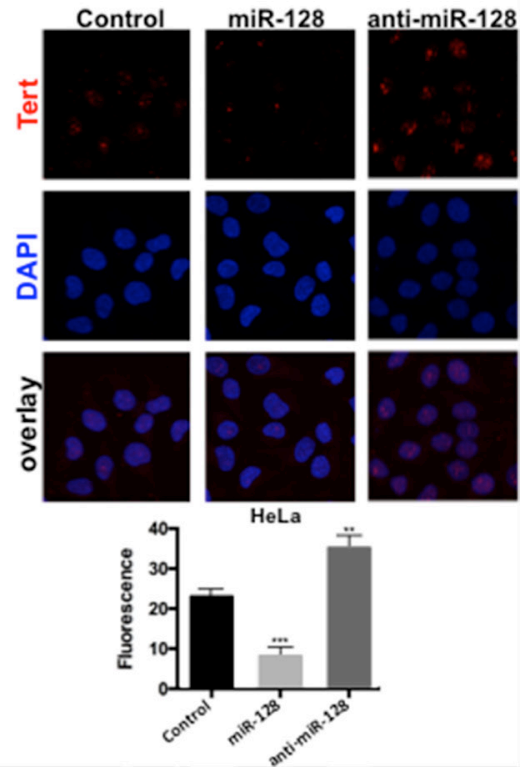

Figure 2: miR-128 reduces the expression of TERT mRNA and protein. (A) miR-modulated HeLa cell lines were generated (over-expressing miR-128, anti-miR-128 or control miR). Relative expression levels of Tert RNA were determined and normalized to beta2-microglobulin (B2M) expression levels. (B) Stably miR-128, anti-miR-128 or control miR control HeLa cells lines were analyzed by western blot analysis using antibodies against Tert and $\alpha$-tubulin. One representative example of three is shown. Quantification of results $(n=3)$ normalized to $\alpha$-tubulin is shown (right panel). (C) Stable miR-128, anti-miR-128 and control miR HeLa cell lines were analyzed by immunofluorescence for Tert expression and co-localization with DAPI. Quantification of results is shown (bottom panel) $(n=3)$. (D) Stable A549, SW620 and PANC1 miR-modulated (miR-128, anti-miR-128 and control miR) cell lines were generated and Western blot analysis were performed using Tert and $\alpha$-tubulin antibodies. Quantification of results $(n=3)$ normalized to $\alpha$-tubulin is shown (bottom panels). All results are shown as mean $\pm \mathrm{SEM}, n=3$, independent biological replicates. ${ }^{*} p<0.05,{ }^{* *} p<0.01,{ }^{* * *} p<0.001$, by two-tailed Student's $t$ test. 
positive control plasmid (as previously described [29, 40]). HeLa cells were co-transfected with one of the three TERT constructs WT Site \#1, WT Site \#2 or the positive control, (Wildtype (WT) seed plasmids) and either miR-128 or miR control mimic oligonucleotides. Luciferase activity was modestly, but significantly reduced in HeLa cells transfected with either one of the two WT TERT constructs and miR-128, relative to miR controls (Figure 3C). As expected, luciferase activity was potently repressed in the positive HeLa cell control (Figure 3C). These experiments supports the conclusion that miR-128 can bind to the predicted binding site likely at both location of the TERT mRNA sequence. Next, we generated a plasmid encoding the TERT binding sites (Site \#2) in which we included mutations in the putative miR-128-binding site of TERT mRNA (Figure 3D, top). HeLa cells co-transfected with the plasmid encoding WT TERT (WT seed plasmid) and miR-128, showed a significant reduced luciferase activity as previously demonstrated (Figure 3C and 3D). In contrast, HeLa cells co-transfected with the mutant TERT mRNA-binding site (Mutated seed plasmid) and either mature miR-128 or control-miR mimics exhibited luciferase activity at similar levels as in the WT TERT and control-miR cells, consistent with miR-128 no longer binding and repressing luciferase reporter-gene expression (Figure 3D). These experiments determine that miR-128 is dependent on the predicted nucleotide sequence to interact with TERT mRNA.

Finally, to test whether miR-128 interacts with TERT mRNA in cells, we isolated Ago complexes containing miRs and target mRNAs by immunopurification from HeLa cells either overexpressing miR-128 or anti-miR128 and assessed relevant complex occupancy by TERT mRNA (Figure 4A). As determined previously (Figure 2A), miR-128 reduces TERT mRNA levels in HeLa cells (Input). In addition, we determined that despite the lower levels of TERT mRNA in HeLa cells (caused by overexpression of miR-128), Ago-bound TERT mRNA was significantly higher in cells overexpressing miR-128 than in cells in which miR-128 was downregulated by antimiR-128 (IP) (Figure 4B, left panel). When correcting for the higher levels of TERT mRNA in HeLa cells treated with anti-miR-128, the difference in bound TERT mRNA was even more significant (IP) (anti-miR-128 corrected) (Figure 4B, left panel). A control, constitutively expressed transcript of the GAPDH gene did not show altered levels of total RNA in cells transduced with miR-128 or anti-miR-128 (Input), or relative differences in Ago immunopurification (IP) (Figure 4B, middle panel). Finally, we verified that TERT mRNA could also be immunopurified by miR-128-Ago in Tera cells, showing that miR-128 significantly reduces TERT mRNA levels in

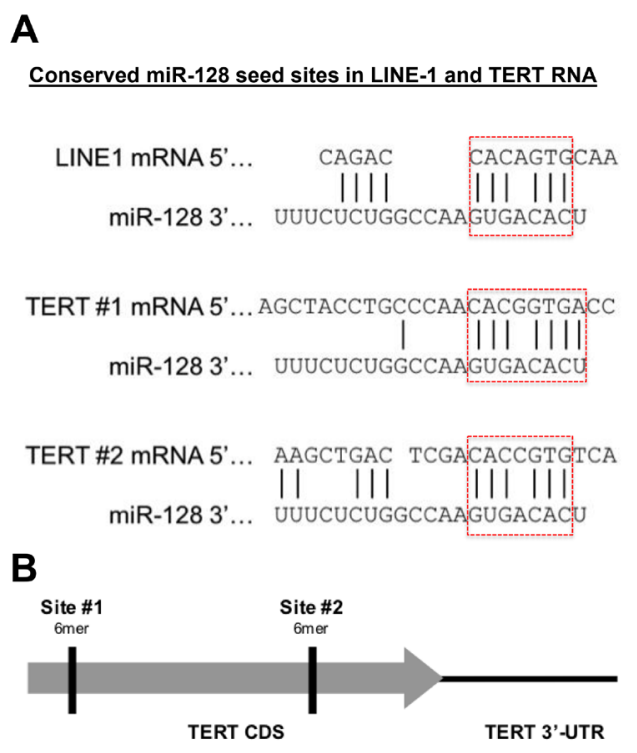

C

D

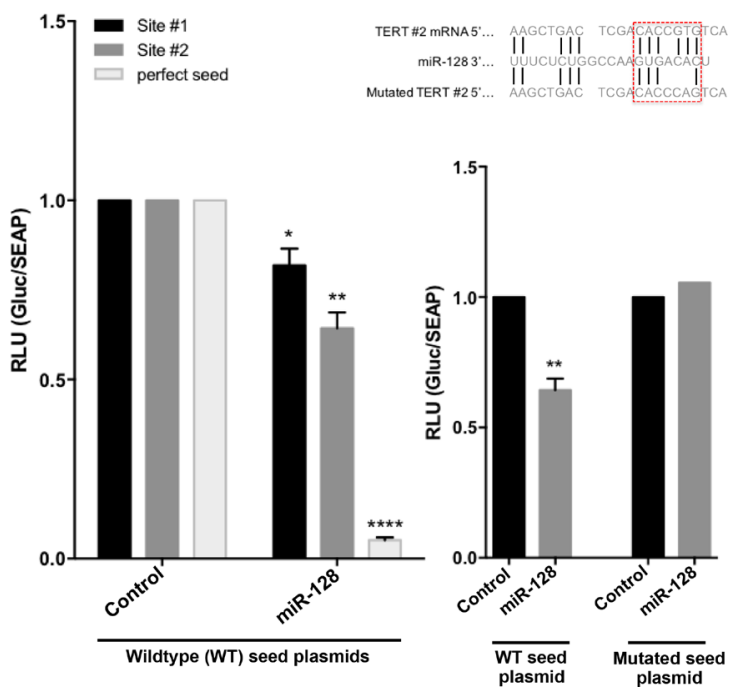

Figure 3: miR-128 interacts with the seed site of the coding sequence of TERT mRNA. (A) Schematic representation of the predicted and functional miR-128 binding sites in long-interspaced element-1 (LINE-1, L1) CDS RNA compared to two predicted miR-128 binding sites in the CDS of TERT mRNA. (B) Schematic representation of the two putative non-perfect 7-mer miR-128 binding sites in the coding sequence of TERT mRNA. (C) Relative luciferase levels of HeLa cell transfected with constructs expressing either TERT binding sequences at site \#1 or site \#2 or a perfect miR-128 seed match (perfect seed) (Wildtype (WT) seed plasmids), along with miR-control (Control) or miR-128 (miR-128) mimics, measured 48 hours post transfection. Results shown as percent change \pm SEM ( $n=3$ independent experiments, $\left.{ }^{*} p<0.05,{ }^{* *} p<0.01,{ }^{* * * *} p<0.0001\right)$ (D) Relative luciferase levels of HeLa cell transfected with constructs expressing the wildtype (WT seed plasmid) or mutated (Mutated seed plasmid) 7-mer TERT binding sequences, along with miR-control (Control) or miR-128 (miR-128) mimics, measured 48 hours post transfection. Results shown as percent change $\pm \operatorname{SEM}(n=3$ independent experiments, $\left.{ }^{* *} p<0.01\right)$. 
Tera cells (Input), and that miR-128 interacts with TERT mRNA in Tera cells, at a significantly manner, when correcting for miR-128 levels (Figure 4B, right panel). This body of work supports the conclusion that miR-128 interacts with TERT mRNA and suggests that the putative miR-128 binding site in the coding region of TERT mRNA is indeed the functional binding site resulting in potent regulation of TERT levels and telomerase activity.

\section{DISCUSSION}

This study is the first to identify that miR-128 targets TERT mRNA and reduces TERT mRNA and protein levels resulting in a decrease in telomerase activity in cancer cells. Telomerase is a cellular reverse transcriptase that maintains chromosome health by extending telomeres and protecting chromosome ends. While telomerase is inactive in most adult cells, it is reactivated in cancer cells allowing continuous proliferation. The necessity for telomerase in continued cancer cell growth makes it an attractive therapeutic target.

microRNAs (miRs) have been established as crucial players in cancer initiation and progression by regulating oncogenes or tumor suppressor genes. miR-128 has previously been found to act as a tumor suppressor and it is downregulated in various types of cancer, including glioma [28], lung cancer [41], prostate cancer [25] and bladder cancer [42]. Furthermore, miR128 is highly enriched in the brain, but not detected in glioma cells. Mechanistically, miR-128 has been found to reduce glioma cell proliferation and promote stem cell self-renewal, by the regulation of the BMI-1 oncogene [28]. miR-128 has also been demonstrated to positively regulate p53 by directly targeting SIRT1, and promote apoptosis in a PUMA-dependent manner [43]. In nonsmall cell lung cancer cells, miR-128 overexpression was observed to suppress invasion and induce cell cycle arrest and apoptosis. Interestingly, when miR-128 was restored, tumorigenicity was greatly suppressed in a mouse model of lung cancer [44]. Taken together, these studies demonstrates that miR-128 functions as a tumor suppressor in many cancer types and our experimental findings add another mechanism by which miR-128 repress the oncogenic phenotype of cancer cells.

The regulation of telomerase activity in healthy cells and in cancer is complex and includes genomic rearrangements, as well as regulation by cellular factors including (but not limited to): Proteinase-activated receptor 1 (PAR1), Telomeric Repeat Factor 1 (TRF1), the Kinase, Endopeptidase and Other Proteins of small Size complex (KEOPS complex) and the Laminin Receptor (LRP) [45-50].

We have previously established that miR-128 interacts with the coding sequence (CDS) of the reverse transcriptase component of long interspersed element 1 (LINE-1) retrotransposons (ORF2), preventing retrotransposition, genomic integration and mutagenesis [29]. Interestingly, the activity of L1 and telomerase is closely intertwined. Specifically, L1 retrotransposons can provide an alternative mechanism to maintain telomere structure in organisms from Drosophila melanogaster to humans and L1 activity has been reported to induce hTERT mRNA levels and telomerase activity in tumor cell lines [51-53].

The finding that the functional miR-128 binding sites are located in both the CDS of telomerase mRNA and the L1 mRNA, responsible for repressing Telomerase activity and L1 mobilization in cancer cells, supports the
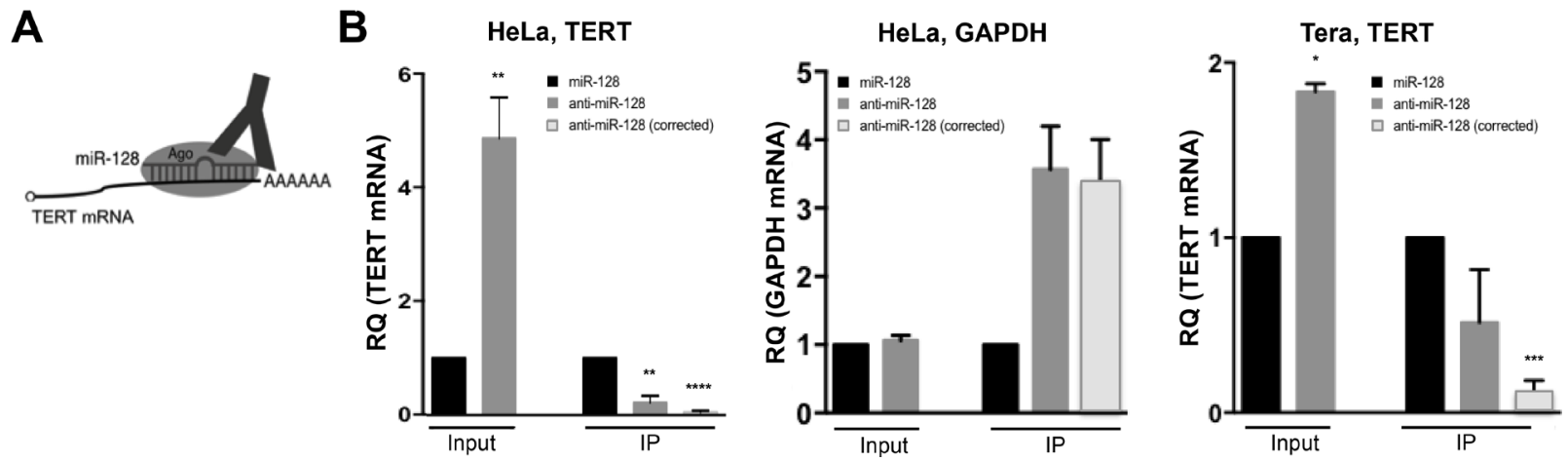

Figure 4: miR-128 directly interacts with TERT mRNA in cells. (A) Schematic of Argonaute immunopurification strategy (AgoRIP) strategy. HeLa or Tera cell lines were generated in which miR-128 was either neutralized or over-expressed (stably transduced with anti-miR-128 or miR-128-expressing constructs). If TERT is a direct target of the miR-128/Ago complex, then Ago immunopurification in cells with neutralized miR-128 will pull out less TERT mRNA compared to miR-128 expressing cells, which will bind TERT mRNA directly. (B) Relative levels of TERT mRNA were determined by q-PCR analysis and (normalized to B2M) in "input samples" of miR modulated HeLa cells. Relative TERT mRNA levels were next determined in IP fractions and normalized to input levels. TERT IP fractions are also shown as "corrected" levels, in which IP TERT levels were corrected for levels of miR-128 in HeLa samples. As a negative control the same samples were analyzed for relative expression of GAPDH levels. Finally, Ago-RIP in Tera cells was performed as described for HeLa cells. Results from 3 independent experiments are shown as mean of IP fraction \pm SEM of three independent experiments $(n=3$, $\left.{ }^{*} p<0.05,{ }^{* *} p<0.01,{ }^{* * *} p<0.001,{ }^{* * * *} p<0.0001\right)$ 
novel concept that one microRNA (for example miR-128) can function by parallel regulation of groups of enzymes (such as RT) by binding to conserved target sequences in the coding region of the enzymes.

\section{MATERIALS AND METHODS}

\section{Cell culture and transductions}

All cells were incubated at $37^{\circ} \mathrm{C}$ and $5 \% \mathrm{CO}_{2}$ and routinely checked for mycoplasma contamination. HeLa cells, which are derived from cervix adenocarcinoma (CCL-2, American Tissue Cell Culture (ATCC)) were cultured in EMEM (SH3024401, Hyclone) supplemented with $10 \%$ FBS. 293 T cells, which are human embryonic kidney (HEK) cells that express and are transformed with large T antigen (CRL-3216, ATCC) were cultured in DMEM (25-501N, Genesee) with 10\% FBS. Tera-1 cells, which originate from metastatic human GCT/teratoma (HTB-105, ATCC) were cultured in McCoy's 5A (16600082, Life Technologies) supplemented with $20 \%$ cosmic serum (SH3008702, Fisher Scientific).

\section{anti-miR library screen}

HeLa cells were transduced with miR Zip Virus Library (MZIPPLVA, System Biosciences), (encoding anti-miRs which neutralizes conserved and/or wellstudied miRs) selected for Puromycin resistance and split to single cell dilutions in 96-well plates. Cells were grown to confluency and telomerase activity was measured using the telomeric repeat amplification protocol (q-TRAP) [9].

\section{Cell transductions}

VSV-G-pseudotyped lentiviral particles were made by transfecting $293 \mathrm{~T}$ cells with $0.67 \mu \mathrm{g}$ of pMD2-G (12259, Addgene), $1.3 \mu \mathrm{g}$ of pCMV-DR8.74 (8455, Addgene) and $2 \mu \mathrm{g}$ of mZIP-miR-128 or mZIPanti-miR-128 using Lipofectamine LTX (15338030, ThermoFisher). Viral supernatants were concentrated using PEG-it (LV810A-1, System Biosciences). Cells were transduced with high titer virus using polybrene (sc134220, Santa Cruz Biotech) and spinoculated at $800 \mathrm{x} g$ at $32^{\circ} \mathrm{C}$ for 30 minutes. Transduced cells were selected and maintained using $10 \mu \mathrm{g} / \mathrm{ml}$ puromycin.

\section{Quantitative telomeric repeat amplification protocol (Q-TRAP)}

HeLa cells were lysed in NP40 Lysis buffer and q-TRAP analysis was carried out. Briefly, lysates were mixed with EGTA (NC9118216), Platinum Taq polymerase (10966034, Life), ACX primer (5'-GCG CGG CTT ACC CTT ACC CTT ACC CTA ACC-3'), TS primer
(5'-AAT CCG TCG AGC AGA GTT-3'), and either SYBR Green Master Mix (4367659, Life) or Forget-Me-Not qPCR Master Mix with Rox (31042-1, Biotium). Samples were incubated for $30 \mathrm{~min}$ at $30^{\circ} \mathrm{C}$ for telomerase extension, then $95^{\circ} \mathrm{C}$ for $10 \mathrm{~min}$ to deactivate telomerase and activate Platinum Taq polymerase, then 40 cycles of $95^{\circ} \mathrm{C}$ for $15 \mathrm{sec}$ and $60^{\circ} \mathrm{C}$ for $1 \mathrm{~min}$. Amplification was normalized to a standard curve of HeLa lysates diluted 1:5 from $1 \mu \mathrm{g} /$ well to $8 \mathrm{ng} /$ well.

\section{RNA extraction and RT-qPCR}

RNA was extracted with Trizol (15596018, ThermoFisher Scientific) according to manufacturer's instructions and cDNA synthesis was performed with the High Capacity Reverse Transcriptase Kit (4368813, Life Technologies). cDNA was amplified relative to GAPDH using the Forget-Me-Not qPCR Master Mix with Rox (31042-1, Biotium) according to the manufacturer's protocol.

\section{Western blot}

Cells were lysed in RIPA buffer (89901, ThermoFisher, Waltham, MA) supplemented with $1 \times$ protease inhibitor cocktail (PI78410, ThermoFisher, Waltham, MA) and then mixed with 4x LDS sample buffer (NP0008, ThermoFisher, Waltham, MA) and boiled at $95^{\circ} \mathrm{C}$ for 10 minutes. Samples were run on NuPAGE Novex 4-12\% Bis-Tris Protein Gels (NP0335, ThermoFisher, ThermoFisher Scientific), and transferred to PVDF membranes. Membranes were incubated overnight at $4 \mathrm{C}$ with rabbit anti-human Tert antibody, (1:100 dilution) (Y182 ab32020, Abcam, Cambridge, MA) then HRP-linked anti-rabbit IgG antibody (1:2000 dilution) (7074S, Cell Signaling Technology, Danvers, MA) and visualized with Pierce ECL Western Blotting Substrate (32106, ThermoFisher, Waltham, MA) on the Bio-Rad ChemiDoc XRS+ System.

\section{Immunofluorescent staining}

Cells were plated on gelatin-coated coverslips, fixed in 4\% paraformaldehyde (Sigma-Aldrich, St. Louis, MO), incubated in blocking buffer ( $1 \%$ bovine serum albumin, 0.3\% Triton X-100 (ThermoFisher, Waltham, MA) in PBS), stained with mouse anti-human Tert antibody incubated overnight at 4C (ab5181, Abcam, Cambridge, MA) at 1:25 dilution, followed by PE-conjugated anti-mouse IgM antibody for 2 hours at room temperature (1:250 dilution) (clone eB121-15F9, eBioscience/ThermoFisher, Waltham, MA). Coverslips were mounted on slides with VectaSheild with DAPI (H-1200, Vector Laboratories, Burlingame, CA) and cells imaged at $63 \mathrm{x}$ on a Zeiss spinning disk confocal microscope. 


\section{Luciferase direct-binding reporter assay}

Wildtype or mutated TERT sequences were cloned into a dual luciferase reporter plasmid (pEZXMT05, Genecopoeia). $3 \times 10^{5} \mathrm{HeLa}$ cells were forwardtransfected with $0.8 \mu \mathrm{g}$ reporter plasmid and $20 \mathrm{nM}$ control mimic or miR-128 mimic with Attractene transfection reagent (301005, Qiagen) according to the manufacturer's instructions. Relative Gaussia luciferase and secreted alkaline phosphatase (SEAP) levels were determined with the Secrete-Pair Dual Luminescence Assay Kit (SPDA-D010, Genecopoeia) on a Tecan Infinite F200 microplate reader.

\section{Argonaute RNA Immunopurifications (AgoRIP)}

Immunopurification of Argonaute from $\mathrm{HeLa}$ and Tera cell extracts was performed using the 4F9 antibody (4F9, Santa Cruz Biotechnology) as described previously [29]. Briefly, $10 \mathrm{~mm}$ plates of $80 \%$ confluent cultured cells were washed with buffer A [ $20 \mathrm{mM}$ Tris$\mathrm{HCl} \mathrm{pH}$ 8.0, $280 \mathrm{mM} \mathrm{KCl}, 10 \mathrm{mM}$ EDTA, $1 \% \mathrm{NP}-40$, $0.2 \%$ Deoxycholate, $2 \mathrm{X}$ Halt protease inhibitor cocktail (Pierce), $200 \mathrm{U} / \mathrm{ml}$ RNaseout (ThermoFisher Scientific) and $1 \mathrm{mM} \mathrm{DTT}$. Protein concentration was adjusted across samples with buffer B $[20 \mathrm{mM}$ Tris- $\mathrm{HCl} \mathrm{pH}$ 8.0, $140 \mathrm{mM} \mathrm{KCl}, 5 \mathrm{mM}$ EDTA pH 8.0, 0.5\% NP-40, $0.1 \%$ deoxycholate, $100 \mathrm{U} / \mathrm{ml}$ Rnaseout (ThermoFisher Scientific), $1 \mathrm{mM}$ DTT and $1 \mathrm{X}$ Halt protease inhibitor cocktail (Pierce)]. Lysates were centrifuged at 16,000xg for $15 \mathrm{~min}$ at $4^{\circ} \mathrm{C}$ and supernatants were incubated with 10-20 $\mu \mathrm{g}$ of 4F9 antibody conjugated to epoxy magnetic beads (M-270 Dynabeads, ThermoFisher) for 2 hours at $4^{\circ} \mathrm{C}$ with gentle rotation. Following magnetic separation, the beads were washed three times five min with $2 \mathrm{ml}$ of buffer C [20 mM Tris-HCl pH 8.0, $140 \mathrm{mM} \mathrm{KCl,} 5$ mM EDTA pH 8.0, $40 \mathrm{U} / \mathrm{ml}$ Rnaseout (ThermoFisher Scientific), $1 \mathrm{mM}$ DTT and $1 \mathrm{X}$ Halt protease inhibitor cocktail (Pierce)]. Following immunopurification, RNA was extracted using miRNeasy kits (217004, Qiagen), following the manufacturer's recommendations and qPCR was performed using custom probes/primers (TERT Forward: ACCAAGCATTCCTGCTCAAG and TERT Reverse: GCTGCTGGTGTCTGCTCTC) and Forget-me-not qPCR master mix (31042-1, Biotium). Results were normalized to their inputs and shown as "corrected" values as a proxy for Ago immunopurification efficiency.

\section{Statistical analysis}

Student's $t$-tests were used to calculate two-tailed $p$ values and data are displayed as mean \pm standard error of the mean (SEM) of independent biological replicates, (n) as indicated.

\section{Abbreviations}

Ago: Argonaute; BMI-1: BMI1 Proto-Oncogene, Polycomb Ring Finger; CDS: coding sequence; GAPDH: Glyceraldehyde 3-phosphate dehydrogenase; GFP: green fluorescent protein; iPSC: induced pluripotent stem cells; KEOPS: the Kinase, Endopeptidase and Other Proteins of small Size; L1 or LINE-1: long-interspaced element-1; LRP: Laminin Receptor; miR: microRNA; miRISC: miR-induced silencing complex; mRNA: messenger; RNA ORF: open reading frame; PAR1: Proteinase-activated receptor 1; PUMA: p53 upregulated modulator of apoptosis; q-TRAP: Real-time quantitative telomeric repeat amplification; RIP: Argonaute-RNA immunopurification; RT: reverse transcriptase; SIRT1: silent mating type information regulation 2 homolog; shRNA: short hairpin RNA; TERT: Telomerase Reverse Transcriptase; TERC: telomerase RNA component; TRF1: Telomeric Repeat Factor 1; WT: wild type.

\section{Author contributions}

KS made the initial identification of miR-128 as a novel telomerase regulator. $\mathrm{HG}, \mathrm{KS}, \mathrm{ES}$ and $\mathrm{AI}$ performed the majority of experiments, with the help of DJ and ID, demonstrating that miR-128 targets TERT mRNA and reduces the levels of Tert mRNA and protein. DGZ performed the Ago RNA IPs and IMP directed all experiments, figure design and wrote the manuscript with help from ID and HG.

\section{CONFLICTS OF INTEREST}

All co-authors implicated in this research approved of this article to be published. No authors have declared any conflicts of interest.

\section{FUNDING}

This work was supported by University of California Cancer Research Coordinating Committee 55205 (IMP), American Cancer Society - Institutional Research Grant 98-279-08 (IMP).

\section{REFERENCES}

1. Hanahan D, Weinberg RA. Hallmarks of cancer: the next generation. Cell. 2011; 144:646-674.

2. Hanahan D, Weinberg RA. The hallmarks of cancer. Cell. 2000; 100:57-70.

3. Greider CW, Blackburn EH. Identification of a specific telomere terminal transferase activity in Tetrahymena extracts. Cell. 1985; 43:405-413. 
4. Levy MZ, Allsopp RC, Futcher AB, Greider CW, Harley CB. Telomere end-replication problem and cell aging. J Mol Biol. 1992; 225:951-960.

5. Morin GB. The human telomere terminal transferase enzyme is a ribonucleoprotein that synthesizes TTAGGG repeats. Cell. 1989; 59:521-529.

6. Low KC, Tergaonkar V. Telomerase: central regulator of all of the hallmarks of cancer. Trends Biochem Sci. 2013; 38:426-434.

7. d'Adda di Fagagna F, Reaper PM, Clay-Farrace L, Fiegler H, Carr P, Von Zglinicki T, Saretzki G, Carter NP, Jackson SP. A DNA damage checkpoint response in telomereinitiated senescence. Nature. 2003; 426:194-198.

8. Shay JW, Bacchetti S. A survey of telomerase activity in human cancer. Eur J Cancer. 1997; 33:787-791.

9. Kim NW, Piatyszek MA, Prowse KR, Harley CB, West MD, Ho PL, Coviello GM, Wright WE, Weinrich SL, Shay JW. Specific association of human telomerase activity with immortal cells and cancer. Science. 1994; 266:2011-2015.

10. Horn S, Figl A, Rachakonda PS, Fischer C, Sucker A, Gast A, Kadel S, Moll I, Nagore E, Hemminki K, Schadendorf D, Kumar R. TERT promoter mutations in familial and sporadic melanoma. Science. 2013; 339:959-961.

11. Huang FW, Hodis E, Xu MJ, Kryukov GV, Chin L, Garraway LA. Highly recurrent TERT promoter mutations in human melanoma. Science. 2013; 339:957-959.

12. Bartel DP. MicroRNAs: genomics, biogenesis, mechanism, and function. Cell. 2004; 116:281-297.

13. Lin PY, Yu SL, Yang PC. MicroRNA in lung cancer. Br J Cancer. 2010; 103:1144-1148.

14. Croce CM. Causes and consequences of microRNA dysregulation in cancer. Nat Rev Genet. 2009; 10:704-714.

15. Kloosterman WP, Plasterk RH. The diverse functions of microRNAs in animal development and disease. Dev Cell. 2006; 11:441-450.

16. Garofalo M, Croce CM. microRNAs: Master regulators as potential therapeutics in cancer. Annu Rev Pharmacol Toxicol. 2011; 51:25-43.

17. Lujambio A, Lowe SW. The microcosmos of cancer. Nature. 2012; 482:347-355.

18. Esquela-Kerscher A, Slack FJ. Oncomirs - microRNAs with a role in cancer. Nat Rev Cancer. 2006; 6:259-269.

19. Johnson SM, Grosshans H, Shingara J, Byrom M, Jarvis R, Cheng A, Labourier E, Reinert KL, Brown D, Slack FJ. RAS is regulated by the let-7 microRNA family. Cell. 2005; 120:635-647.

20. Hrdlickova R, Nehyba J, Bargmann W, Bose HR Jr. Multiple tumor suppressor microRNAs regulate telomerase and TCF7, an important transcriptional regulator of the Wnt pathway. PLoS One. 2014; 9:e86990.

21. Mitomo S, Maesawa C, Ogasawara S, Iwaya T, Shibazaki M, Yashima-Abo A, Kotani K, Oikawa H, Sakurai E, Izutsu N, Kato K, Komatsu H, Ikeda K, et al. Downregulation of miR-138 is associated with overexpression of human telomerase reverse transcriptase protein in human anaplastic thyroid carcinoma cell lines. Cancer Sci. 2008; 99:280-286.

22. Li M, Fu W, Wo L, Shu X, Liu F, Li C. miR-128 and its target genes in tumorigenesis and metastasis. Experimental cell research. 2013; 319:3059-3064.

23. Shen L, Chen XD, Zhang YH. MicroRNA-128 promotes proliferation in osteosarcoma cells by downregulating PTEN. Tumour Biology. 2014; 35:2069-2074.

24. Wu L, Shi B, Huang K, Fan G. MicroRNA-128 suppresses cell growth and metastasis in colorectal carcinoma by targeting IRS1. Oncol Rep. 2015; 34:2797-2805.

25. Khan AP, Poisson LM, Bhat VB, Fermin D, Zhao R, Kalyana-Sundaram S, Michailidis G, Nesvizhskii AI, Omenn GS, Chinnaiyan AM, Sreekumar A. Quantitative proteomic profiling of prostate cancer reveals a role for miR-128 in prostate cancer. MCP. 2010; 9:298-312.

26. Cui JG, Zhao Y, Sethi P, Li YY, Mahta A, Culicchia F, Lukiw WJ. Micro-RNA-128 (miRNA-128) downregulation in glioblastoma targets ARP5 (ANGPTL6), Bmi-1 and E2F-3a, key regulators of brain cell proliferation. J Neurooncol. 2010; 98:297-304.

27. Hauser B, Zhao Y, Pang X, Ling Z, Myers E, Wang P, Califano J, Gu X. Functions of MiRNA-128 on the regulation of head and neck squamous cell carcinoma growth and apoptosis. PLoS One. 2015; 10:e0116321.

28. Godlewski J, Nowicki MO, Bronisz A, Williams S, Otsuki A, Nuovo G, Raychaudhury A, Newton HB, Chiocca EA, Lawler S. Targeting of the Bmi-1 oncogene/ stem cell renewal factor by microRNA-128 inhibits glioma proliferation and self-renewal. Cancer Res. 2008; 68:9125-9130.

29. Hamdorf M, Idica A, Zisoulis DG, Gamelin L, Martin C, Sanders KJ, Pedersen IM. miR-128 represses L1 retrotransposition by binding directly to L1 RNA. Nat Struct Mol Biol. 2015; 22:824-831.

30. Idica A, Sevrioukov EA, Zisoulis DG, Hamdorf M, Daugaard I, Kadandale P, Pedersen IM. MicroRNA miR128 represses LINE-1 (L1) retrotransposition by downregulating the nuclear import factor TNPO1. J Biol Chem. 2017; 292:20494-20508.

31. Scott EC, Gardner EJ, Masood A, Chuang NT, Vertino PM, Devine SE. A hot L1 retrotransposon evades somatic repression and initiates human colorectal cancer. Genome Res. 2016; 26:745-755.

32. Shukla R, Upton KR, Munoz-Lopez M, Gerhardt DJ, Fisher ME, Nguyen T, Brennan PM, Baillie JK, Collino A, Ghisletti S, Sinha S, Iannelli F, Radaelli E, et al. Endogenous retrotransposition activates oncogenic pathways in hepatocellular carcinoma. Cell. 2013; 153:101-111.

33. Tubio JM, Li Y, Ju YS, Martincorena I, Cooke SL, Tojo M, Gundem G, Pipinikas CP, Zamora J, Raine K, Menzies A, Roman-Garcia P, Fullam A, et al. Mobile DNA in cancer. Extensive transduction of nonrepetitive DNA mediated by L1 retrotransposition in cancer genomes. Science. 2014; $345: 1251343$. 
34. Lee E, Iskow R, Yang L, Gokcumen O, Haseley P, Luquette LJ 3rd, Lohr JG, Harris CC, Ding L, Wilson RK, Wheeler DA, Gibbs RA, Kucherlapati R, et al. Landscape of somatic retrotransposition in human cancers. Science. 2012; 337:967-971.

35. Rodic N, Sharma R, Sharma R, Zampella J, Dai L, Taylor MS, Hruban RH, Iacobuzio-Donahue CA, Maitra A, Torbenson MS, Goggins M, Shih Ie M, Duffield AS, et al. Long interspersed element-1 protein expression is a hallmark of many human cancers. Am J Pathol. 2014; 184:1280-1286.

36. Rodic N, Steranka JP, Makohon-Moore A, Moyer A, Shen P, Sharma R, Kohutek ZA, Huang CR, Ahn D, Mita P, Taylor MS, Barker NJ, Hruban RH, et al. Retrotransposon insertions in the clonal evolution of pancreatic ductal adenocarcinoma. Nat Med. 2015; 21:1060-1064.

37. Miki Y, Nishisho I, Horii A, Miyoshi Y, Utsunomiya J, Kinzler KW, Vogelstein B, Nakamura Y. Disruption of the APC gene by a retrotransposal insertion of L1 sequence in a colon cancer. Cancer research. 1992; 52:643-645.

38. Pedersen IM, Cheng G, Wieland S, Volinia S, Croce CM, Chisari FV, David M. Interferon modulation of cellular microRNAs as an antiviral mechanism. Nature. 2007; 449:919-922.

39. Nathans R, Chu CY, Serquina AK, Lu CC, Cao H, Rana TM. Cellular microRNA and P bodies modulate host-HIV-1 interactions. Mol Cell. 2009; 34:696-709.

40. Daugaard I, Sanders KJ, Idica A, Vittayarukskul K, Hamdorf M, Krog JD, Chow R, Jury D, Hansen LL, Hager H, Lamy P, Choi CL, Agalliu D, et al. miR-151a induces partial EMT by regulating E-cadherin in NSCLC cells. Oncogenesis. 2017; 6:e366.

41. Tafsiri E, Darbouy M, Shadmehr MB, Zagryazhskaya A, Alizadeh J, Karimipoor M. Expression of miRNAs in non-small-cell lung carcinomas and their association with clinicopathological features. Tumour Biology. 2015; 36:1603-1612.

42. Zhao D, Han W, Liu X, Cui D, Chen Y. MicroRNA-128 promotes apoptosis in lung cancer by directly targeting NIMA-related kinase 2. Thorac Cancer. 2017; 8:304-311.

43. Adlakha YK, Saini N. miR-128 exerts pro-apoptotic effect in a p53 transcription-dependent and -independent manner via PUMA-Bak axis. Cell Death Dis. 2013; 4:e542.

44. Hu J, Cheng Y, Li Y, Jin Z, Pan Y, Liu G, Fu S, Zhang Y, Feng K, Feng Y. microRNA-128 plays a critical role in human non-small cell lung cancer tumourigenesis, angiogenesis and lymphangiogenesis by directly targeting vascular endothelial growth factor-C. Eur J Cancer. 2014; 50:2336-2350.

45. Peifer M, Hertwig F, Roels F, Dreidax D, Gartlgruber M, Menon R, Kramer A, Roncaioli JL, Sand F, Heuckmann JM, Ikram F, Schmidt R, Ackermann S, et al. Telomerase activation by genomic rearrangements in high-risk neuroblastoma. Nature. 2015; 526:700-704.

46. Krauskopf A, Blackburn EH. Control of telomere growth by interactions of RAP1 with the most distal telomeric repeats. Nature. 1996; 383:354-357.

47. van Steensel B, de Lange T. Control of telomere length by the human telomeric protein TRF1. Nature. 1997; 385:740-743.

48. Downey M, Houlsworth R, Maringele L, Rollie A, Brehme M, Galicia S, Guillard S, Partington M, Zubko MK, Krogan NJ, Emili A, Greenblatt JF, Harrington L, et al. A genome-wide screen identifies the evolutionarily conserved KEOPS complex as a telomere regulator. Cell. 2006; 124:1155-1168.

49. Lin SY, Elledge SJ. Multiple tumor suppressor pathways negatively regulate telomerase. Cell. 2003; 113:881-889.

50. Naidoo K, Malindisa ST, Otgaar TC, Bernert M, Da Costa Dias B, Ferreira E, Reusch U, Knackmuss S, Little M, Weiss SF, Letsolo BT. Knock-Down of the $37 \mathrm{kDa} / 67 \mathrm{kDa}$ Laminin Receptor LRP/LR Impedes Telomerase Activity. PloS One. 2015; 10:e141618.

51. Biessmann H, Valgeirsdottir K, Lofsky A, Chin C, Ginther B, Levis RW, Pardue ML. HeT-A, a transposable element specifically involved in "healing" broken chromosome ends in Drosophila melanogaster. Mol Cell Biol. 1992; 12:3910-3918.

52. Kopera HC, Moldovan JB, Morrish TA, Garcia-Perez JL, Moran JV. Similarities between long interspersed element-1 (LINE-1) reverse transcriptase and telomerase. Proceedings of the National Academy of Sciences of the United States of America. 2011; 108:20345-20350.

53. Aschacher T, Wolf B, Enzmann F, Kienzl P, Messner B, Sampl S, Svoboda M, Mechtcheriakova D, Holzmann $\mathrm{K}$, Bergmann M. LINE-1 induces hTERT and ensures telomere maintenance in tumour cell lines. Oncogene. 2016; 35:94-104. 